\title{
Feedforward Pitch Control Using Wind Speed Estimation
}

\author{
Yoonsu Nam ${ }^{*}$ Jeonggi Kim ${ }^{\dagger}$, Insu Paek*, Young-Hwan Moon**, Seog-Joo Kim**, and Dong-Joon Kim** \\ $\dagger^{*}$ Dept. of Mechanical and Mechatronics Eng., Kangwon National University, Chunchon, Korea \\ ** Smart Grid Research Division, Korea Electrotechnology Research Institute, Changwon, Korea
}

\begin{abstract}
The dynamic response of a multi-MW wind turbine to a sudden change in wind speed is usually slow, because of the slow pitch control system. This could cause a large excursion of the rotor speed and an output power over the rated. A feedforward pitch control can be applied to minimize the fluctuations of these parameters. This paper introduces the complete design steps for a feedforward pitch controller, which consist of three stages, i.e. the aerodynamic torque estimation, the 3-dimensional lookup table for the wind seed estimation, and the calculation of the feedforward pitch amount. The effectiveness of the feedforward control is verified through numerical simulations of a multi-MW wind turbine.
\end{abstract}

Key Words: Feedforward control, Pitch control, Power regulation, Wind turbine, Wind speed estimation

\section{INTRODUCTION}

A wind turbine is an energy conversion device. Maximizing the energy capture from the wind is an important design criterion for wind turbines. Another important issue is the minimization of the mechanical loads of the blades, the drive train and the tower. Nowadays, most multi-MW wind turbines apply variable speed and variable pitch (VSVP) technology to meet these design criteria [1], [2]. The efficiency of the energy conversion is usually represented by the power coefficient, $\mathrm{Cp}$. The ideal maximum of the power coefficient is $16 / 27=0.593$, which is known as the Betz limit [3]. There are two control variables for wind turbine operation, which are the generator reaction torque and the blade pitch. In the below rated wind speed region, the wind turbine is operated so as to extract the maximum energy from the wind, i.e. the max-Cp operation. To maintain the max-Cp condition, the rotor speed should be changed proportionally to the wind speed using the generator torque control, while the blade pitch is set to a fixed position, $\beta_{0}$. However, in the above rated wind speed region, the power coefficient $\mathrm{Cp}$ is controlled away from the max-Cp condition. It is controlled to be inversely proportional to the third power of the wind speed by the blade pitch control. Usually, in this wind speed region, the generator torque is maintained at the rated torque.

The dynamic response of a multi-MW wind turbine to wind turbulence is slow. In the above rated wind speed region, where

\footnotetext{
Manuscript received Oct. 25, 2010; revised Jan. 24, 2011

TCorresponding Author: jeonggi@kangwon.ac.kr

Tel: +82-33-253-0840, Fax: $+82-2-878-1452$, Kangwon Nat'1 Univ.

* Dept. of Mechanical and Mechatronics Eng., Kangwon National Univertisy, Korea

** Smart Grid Research Division, Korea Eletrotechnology Research Institute, Korea
}

the blade pitch is controlled to maintain the rotor speed at its rated level, it usually takes more than 2 second for the rotor speed to reach the steady state for a step change in wind speed.

This is a consequence of the huge moment of inertia in the rotor. The rotor speed can not change instantaneously even though there is sudden increase in an aerodynamic torque due to a step change in the wind speed. The pitch actuator starts to engage in the control action only after the rotor speed error is developed. The slow reaction of the pitch control system results in large fluctuations in the rotor speed, which make the quality of the power regulation problematic. The application of feedforward pitch control can improve this situation. If the wind speed can be estimated, then the amount of feedforward pitch can be calculated based on this information [4]. The feedforward pitch generates a time-ahead pitching command to the wind turbine unlike the conventional PI pitch control strategy which relies only on the rotor speed feedback. The feedforward pitch control decreases the rotor speed fluctuations, which might also be advantageous in alleviating the blade mechanical loads, and in enhancing the power regulation performance of the wind turbine.

This paper deals with the feedforward pitch controller design and its verification through numeric simulations. The proposed feedforward control differs from that of Hooft et al. [4] in the control law structure of the feedforward mechanism. This paper proposes a feedforward pitch demand system, while the design problem of Hooft et al. was for a feedforward pitch rate demand system. A drive train model of a multiMW wind turbine is used for this analysis. A baseline VSVP controller should be pre-designed prior to the application of the feedforward pitch control. The performance of the feedforward control is evaluated with a baseline VSVP control system. 


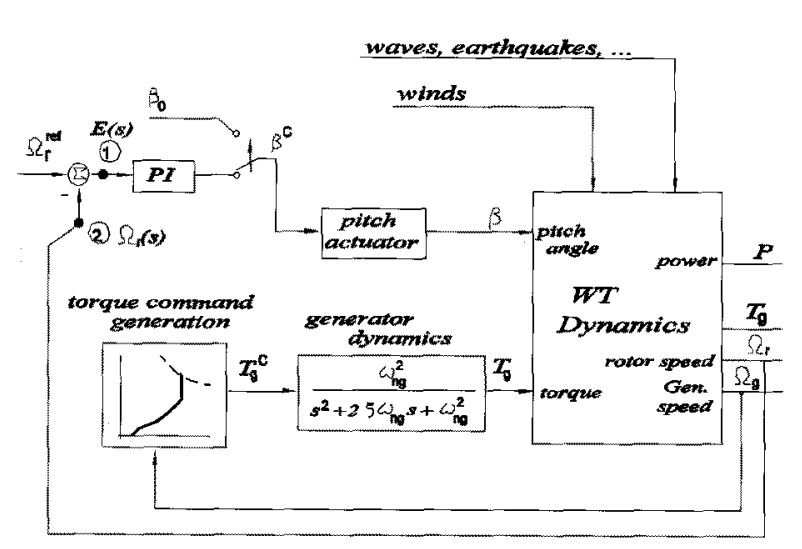

Fig. 1. Schematic of a VSVP control system.

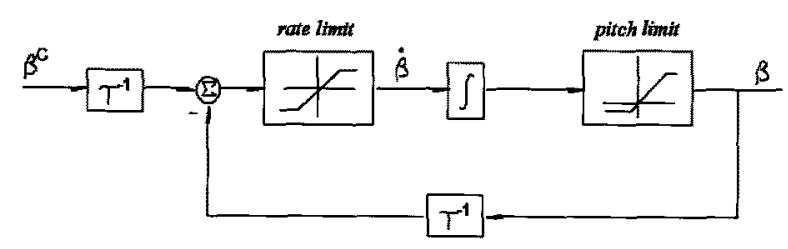

Fig. 2. Model of a pitch actuator.

\section{DRive Train Model and VSVP CONTROLleR DESIGN}

Fig. 1 shows the structure of a VSVP control system. There are two feedback loops, which are the generator torque and blade pitch control loops.

\section{A. Modeling of the generator, the pitch actuator, and the wind turbine dynamic}

Back-to-back converters connected through a DC capacitor control the generator torque as well as manage the active and reactive power of the generator. The generator side converter is in charge of the torque control. A q-axis rotor current for a doubly fed induction generator (DFIG) or a q-axis stator current for a permanent magnet synchronous generator (PMSG) is controlled for this purpose [5]-[8]. From the point of view of the control system design of a wind turbine, it is sufficient to model only the generator side converter. In general, the following second order dynamic system is used to represent the overall behavior of the generator torque control action by the generator side converter.

$$
\frac{T_{g}(s)}{T_{g}^{C}(s)}=\frac{\omega_{n g}^{2}}{s^{2}+2 \varsigma_{n g} \omega_{n g} s+\omega_{n g}^{2}} .
$$

In the above equation, $T_{g}^{C}$ is the generator torque command, $\omega_{n g}(\sim 40 \mathrm{r} / \mathrm{s})$ is the natural frequency and $\zeta_{n g}(\sim 0.7)$ is the damping ratio of the generator dynamics [9]. The blade pitch angle is actuated by an electric motor or a hydraulic actuator which can be modelled as:

$$
\frac{\beta(s)}{\beta^{C}(s)}=\frac{1}{1+\tau_{p} s}
$$

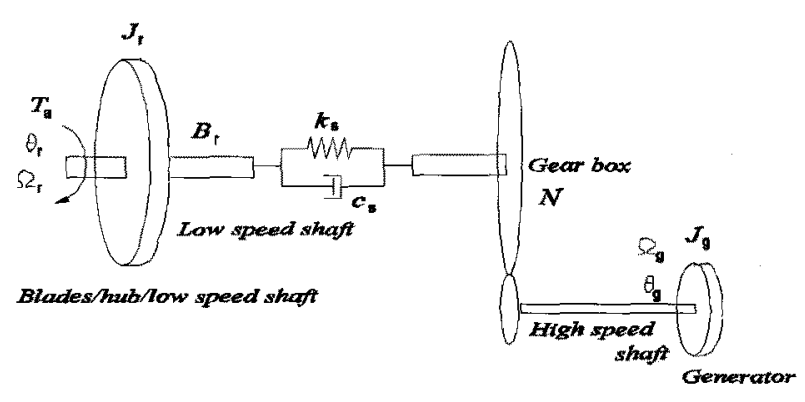

Fig. 3. A drive train model.

where $\beta^{C}$ is the pitch angle demand and $\tau_{p}(\sim 0.04 \mathrm{r} / \mathrm{s})$ is the time constant of the pitch actuator. It is necessary and important for a realistic simulation to include the saturation characteristic in the actuator travel and its rate as depicted in Fig. 2. In general, the pitch ranges from $-3^{\circ}$ to $90^{\circ}$ and maximum pitch rates of $\pm 10^{\circ} / \mathrm{s}$ are typical values for a multiMW wind turbine [2].

The block of 'WT Dynamics' in Fig. 1 represents a dynamic model of a wind turbine. Sophisticated computer codes are required to exactly simulate the wind turbine dynamics. However, the drive train model in Fig. 3 is sufficient for a VSVP control system design [10]. The governing equation motion of this model is given by:

$$
\begin{gathered}
J_{r} \frac{d \Omega_{r}}{d t}=T_{a}-k_{S}\left(\theta_{r}-\frac{1}{N} \theta_{g}\right)-c_{S}\left(\Omega_{r}-\frac{1}{N} \Omega_{g}\right)-B_{r} \Omega_{r} \\
J_{g} \frac{d \Omega_{g}}{d t}=\frac{k_{S}}{N}\left(\theta_{r}-\frac{1}{N} \theta_{g}\right)+\frac{c_{S}}{N}\left(\Omega_{r}-\frac{1}{N} \Omega_{g}\right)-B_{g} \Omega_{g}-T_{g} .
\end{gathered}
$$

The parameters used in Eq. (3) are summarized in Table I. $T_{a}$, which represents the aerodynamic torque developed by the rotor and the wind speed, is given by:

$$
T_{a}=\frac{P}{\Omega_{r}}=\frac{1}{2} \rho \pi R^{2} \frac{C_{P}(\lambda, \beta)}{\Omega_{r}} v^{3}=\frac{1}{2} \rho \pi R^{3}\left\{\frac{C_{P}(\lambda, \beta)}{\lambda}\right\} v^{2}
$$

where $P$ is the electric power, $R$ is the rotor radius, and $C_{P}$ is the power coefficient of the wind turbine. As shown in Eq. (4), $C_{P}$ is a function of the tip speed ratio $\lambda\left(=R \Omega_{r} / v\right)$ and the pitch angle $\beta$.

\section{B. VSVP control system design}

As explained in the beginning of this section, the generator torque is controlled by the high speed switching power electronics of the generator side converter. The operation of this torque control is modeled as a second order dynamic of Eq. (1). Therefore, as shown in Fig. 1, the torque control loop is closed with the inclusion of a generator torque command block, which is a lookup table for the generator torque as a function of the generator speed. Considering the mechanical losses through the drive train, the generator torque schedule over the turbulent wind should be designed to ensure the max$C_{P}$ operation in the below rated wind speed region. It must also be designed to achieve a uniform rated power output in the above rated wind speed region.

The pitch control loop design is accomplished by selecting the PI gains depicted in Fig. 1. Even though this looks simple, it is not so straight forward because of the nonlinear behavior of the wind turbine dynamics. Moreover, the pitch 
TABLE I

PARAMETERS OF A DRIVE TRAIN MODEL

\begin{tabular}{|c|c|c|c|}
\hline Symbol & Description & Unit & Value \\
\hline$J_{r}$ & Inertia of three blades, hub and low speed shaft & $\mathrm{Kgm}^{2}$ & 8274802 \\
\hline$J_{g}$ & Inertia of generator & $\mathrm{Kgm}$ & 64000 \\
\hline$B_{r}$ & Damping of low speed shaft & $\mathrm{Nm} /(\mathrm{r} / \mathrm{s})$ & 32000 \\
\hline$B_{g}$ & Damping of high speed shaft & $\mathrm{Nm} /(\mathrm{r} / \mathrm{s})$ & 0 \\
\hline$k_{s}$ & Torsional stiffness of drive train axis & $\mathrm{Nm} / \mathrm{r}$ & 33158077345 \\
\hline$c_{s}$ & Torsional damping of drive train axis & $\mathrm{Nm} /(\mathrm{r} / \mathrm{s})$ & 4588932 \\
\hline$N$ & Gear ratio & - & 1 \\
\hline$T_{g}$ & Generator reaction torque & $\mathrm{Nm}$ & - \\
\hline$\Omega_{g}$ & Generator speed & $r$ & - \\
\hline$\theta_{r}$ & Rotor rotational angle & $\mathrm{r}$ & - \\
\hline$\theta_{g}$ & Generator rotational angle & $\mathrm{reg} / \mathrm{rpm}$ & - \\
\hline$k_{P}$ & Proportional gain & $d e g / r p m / s$ & 2.292 \\
\hline$k_{I}$ & Integral gain & & 1.833 \\
\hline
\end{tabular}

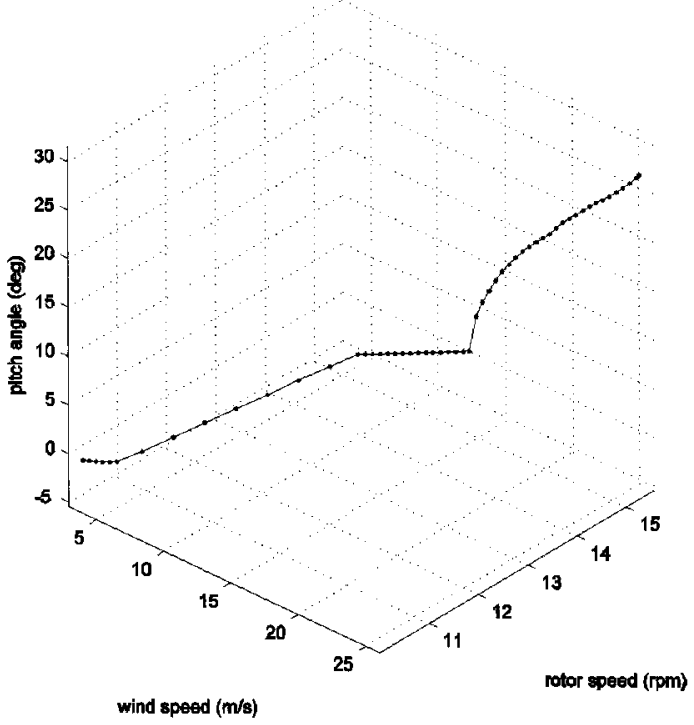

Fig. 4. Operating points for a multi-MW wind turbine.

loop bandwidth, which is almost the same as the crossover frequency of the pitch loop gain transfer function, should be set appropriately for the target wind turbine. The pitch loop bandwidth is to be set high enough to extract the wind energy from the turbulent wind, but not so high as to make the wind turbine operation unstable [11].

The pitch loop control system design starts by acquiring linearized dynamics for the operating points of the wind turbine. The operating point is a set of parameters, i.e. ( $v$, $\beta, \Omega_{r}$ ) which completely specifies the steady state operation of the wind turbine. The relation between these parameters under the steady state condition can be obtained from Eq. (3), which is given by:

$$
\begin{aligned}
& \frac{T_{a}}{N}-\frac{B_{r} \Omega_{r}}{N}-B_{g} \Omega_{g}-T_{g} \\
& =\frac{1}{2 N} \rho \pi R^{3}\left\{\frac{C_{P}(\lambda, \beta)}{\lambda}\right\} v^{2}-\frac{B_{r} \Omega_{r}}{N}-N B_{g} \Omega_{r}-T_{g}=0 .
\end{aligned}
$$

Depending on the wind speed region, either the pitch, $\beta$, or the rotor speed, $\Omega_{r}$, is fixed. Therefore, for a given wind speed, $v$, a set of these three parameters which satisfies the steady state algebraic relation in Eq. (5) can be sought out. Fig. 4 shows a sample plot of the steady state operating points for a multi-MW wind turbine. An analysis of the open loop
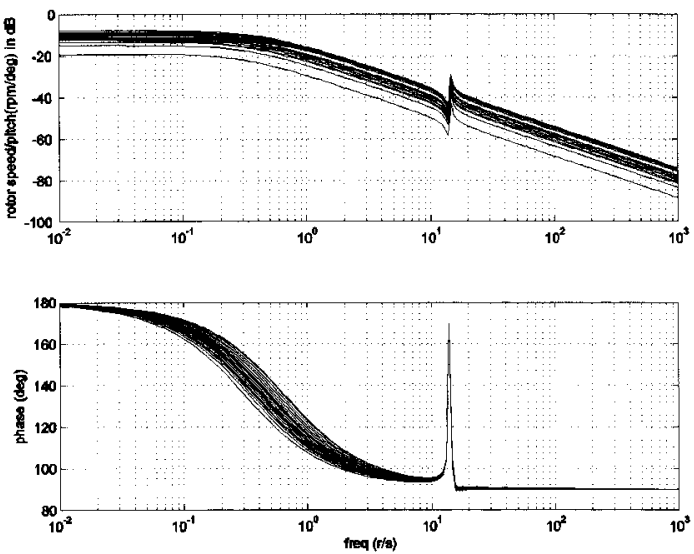

Fig. 5. Frequency responses of the rotor speed for the input of pitch at the operating conditions of the above rated wind speeds.

dynamics at these operating points is crucial to the PI pitch loop design. Fig. 5 shows the frequency responses of the rotor speed (rpm) for the pitch input (deg). They are for a multi-MW wind turbine and are obtained from the linearized dynamics at the operating points of Fig. 4. Overall, the rotor speed output dynamics for the pitch input behave like a first-order system. However, the DC gains vary a great deal depending on the operating conditions (i.e. wind speeds). The variation of these gains originates from the pitch effectiveness difference for each operating point, which is explained in the following.

The pitch effectiveness, which is defined as $\left(\partial T_{a} / \partial \beta\right)_{o}$, represents the variation of the aerodynamic torque for an infinitesimal change in pitch angle. It has a negative sign, which means that the aerodynamic torque decreases with an increase in pitch. Furthermore, the magnitude of this parameter gets larger as the wind speed increases. The above findings hint at which procedures are required for the PI pitch loop design. The first step is to determine the PI gains for any operating point which satisfies the pitch loop bandwidth requirement. The next step is to set the gain scheduling parameter, $k_{G}(\beta)$, which is defined as:

$$
k(s)=k_{P}+k_{I} / s \Rightarrow k(s)=k_{G}(\beta)\left(k_{P}+k_{I} / s\right) .
$$

The necessity of gain scheduling in the pitch loop PI controller comes from the variation of the pitch effectiveness over the wind speed. Fig. 6 shows an example of the gain variation of $k_{G}(\beta)$, scheduled with the pitch angle to compensate for 


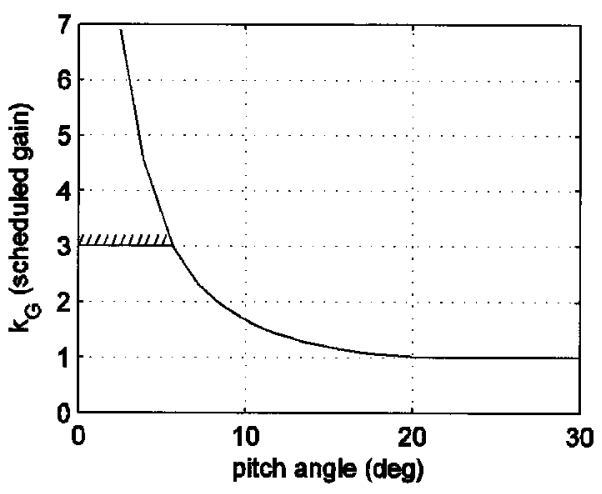

Fig. 6. Scheduled gain variation as a function of pitch.

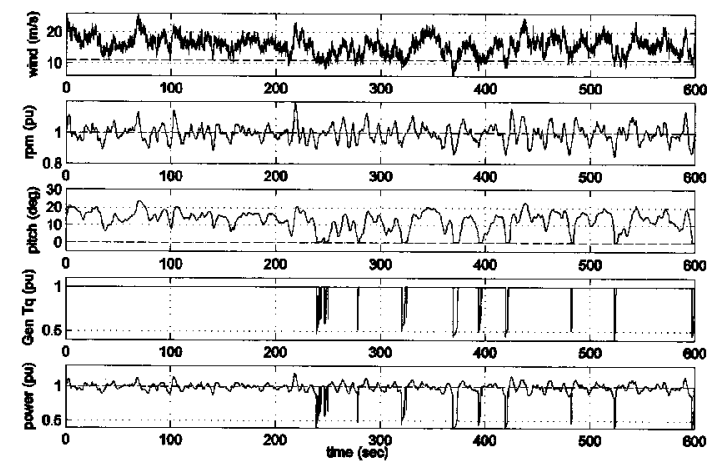

Fig. 7. Simulation results showing a PI pitch loop design for a multi-MW wind turbine.

the above mentioned pitch effectiveness variation. Note that a fairly high scheduled gain is required at a low pitch angle (i.e. in the rated wind speed region).

This section ends by introducing the simulation results for a multi-MW wind turbine, which are shown in Fig. 7. The PI gains are selected to have a pitch loop crossover frequency of $1 \mathrm{r} / \mathrm{s}$ with a gain scheduling similar to that of Fig. 6. The first window of this figure shows the hub height wind used in the simulation. It has a mean wind speed of $16 \mathrm{~m} / \mathrm{s}$ and a turbulence intensity (TI) of $18 \%$. The dashed line represents the rated wind speed for this machine. The second window is the rotor speed in units of PU. Because of high turbulence, there are some fluctuations in the rotor speed at around its rated limit. The third window shows the variation of the pitch. Notice that it hits and stays at $\beta_{0}=0^{\circ}$, when the wind is below the rated wind speed. The fourth window is the generator torque in units of pu. The torque drops rapidly for $\beta_{0}=0^{\circ}$ because of the vertical torque schedule, which is similar to the one shown in Fig. 1. The final plot is the output power in units of PU. Because the torque is controlled to the rated value in the above rated wind speed region, there exists the same pattern of fluctuations in the power as in the rotor speed. The minimization of these fluctuations by applying feedforward control is the main theme of the next section.

\section{Feedforward Pitch Controller Design}

As explained above, the pitch control action is slow. Therefore, a large excursion of the rotor speed is developed when there is a sudden increase in wind speed. This can be noticed in the time domain responses at around 215 seconds in Fig. 7.
By applying feedforward control, these excursions can be minimized. Fig. 8 shows a schematic diagram of the feedforward control algorithm, which is composed of three consecutive processing modules. The first module in a feedforward pitch control is the aerodynamic torque estimation. The second is the estimation of the wind speed through a 3-dimensional lookup table. The final stage determines the amount of feedforward control using two feedforward gains.

The governing equations of the drive train model, i.e. Eq (3), can be combined into one equation as:

$$
J_{t} \frac{d \Omega_{r}}{d t}=T_{a}-N T_{g}-T_{L}
$$

where $J_{t}=J_{r}+N^{2} J_{g}$ and $T_{L}$ represent all of the mechanical losses including $B_{r} \Omega_{r}$ term in Eq. (3). Augmenting the unknown $T_{a}$ in the state vector, the above equation can be rearranged into the state space form as:

$$
\begin{aligned}
\dot{x} & =\left\{\begin{array}{c}
\dot{\Omega}_{r} \\
\dot{T}_{a}
\end{array}\right\}=\left[\begin{array}{cc}
0 & 1 / J_{t} \\
0 & 0
\end{array}\right]\left\{\begin{array}{c}
\Omega_{r} \\
T_{a}
\end{array}\right\} \\
& +\left(\begin{array}{c}
-N / J_{t} \\
0
\end{array}\right) T_{g}+\left(\begin{array}{c}
-1 / J_{t} \\
0
\end{array}\right) T_{L}+\left(\begin{array}{c}
-N / J_{t} \\
N
\end{array}\right) w_{g} \\
y & =\left(\begin{array}{ll}
1 & 0
\end{array}\right)\left\{\begin{array}{l}
\Omega_{r} \\
T_{a}
\end{array}\right\}+v
\end{aligned}
$$

where $w_{g}$ and $v$ represent the input process and the output sensor noise. A Kalman filter is applied to estimate the aerodynamic torque, which has the structure of:

$$
\begin{aligned}
\dot{\hat{x}} & =\left\{\begin{array}{c}
\dot{\hat{\Omega}}_{r} \\
\dot{\hat{T}}_{a}
\end{array}\right\}=\left[\begin{array}{cc}
0 & 1 / J_{t} \\
0 & 0
\end{array}\right]\left\{\begin{array}{l}
\hat{\Omega}_{r} \\
\hat{T}_{a}
\end{array}\right\} \\
& +\left(\begin{array}{c}
-N / J_{t} \\
0
\end{array}\right) T_{g}+\left(\begin{array}{c}
-1 / J_{t} \\
0
\end{array}\right) T_{L}+L\left(\Omega_{r}-\hat{\Omega}_{r}\right)
\end{aligned}
$$

where variables with that are to be estimated and $L$ is the Kalman filter gain [12-13].

The next step of the feedforward control is to build a 3dimensional lookup table, which outputs the estimated wind speed for the input of the estimated aerodynamic torque and two measurable variables, i.e. the pitch and the rotor speed. This table can be constructed by using Eq. (4), which is reformulated in the form of:

$$
\hat{T}_{a}=\frac{1}{2} \rho \pi R^{3}\left\{\frac{C_{P}(\hat{\lambda}, \beta)}{\hat{\lambda}}\right\} \hat{v}^{2}
$$

where $\hat{\lambda}\left(=R \Omega_{r} / \hat{v}\right)$ is the estimated tip speed ratio. The above relationship consists of 4 variables. Because the pitch and rotor speed are measurable and the aerodynamic torque can be estimated by Eq. (9), the wind velocity is the only unknown and it can be estimated by numerically solving Eq. (10).

The final step of the feedforward controller design is to determine the two feedforward gains of $(\partial \beta / \partial v)_{0}$ and $\left(\partial \beta / \partial \Omega_{r}\right)_{o}$ as shown in Fig. 8. In order for a wind turbine to generate the rated power in the above rated wind speed region, a set of variables, i.e. $\left(v, \Omega_{r}, \beta\right)$, should meet the relation of:

$$
P=\frac{1}{2} \pi R^{2} C_{P}(\lambda, \beta) v^{3}=P_{\text {rated }}=\text { constant }
$$




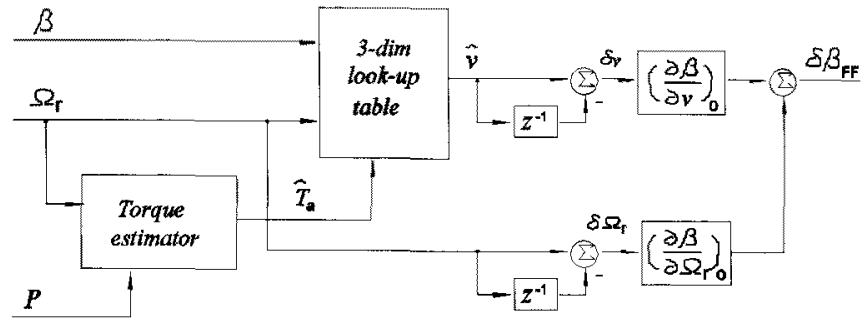

Fig. 8. Schematic of a feedforward pitch control.

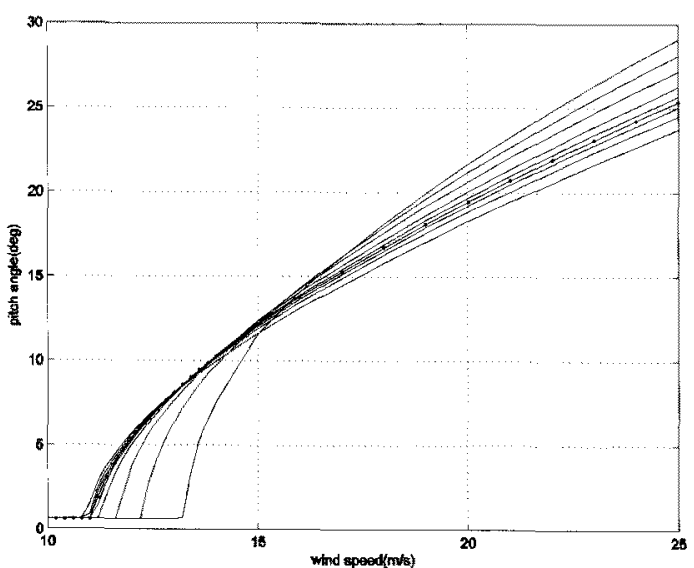

Fig. 9. Plot of pitch and wind speed for 8 different rotor speed producing the rated power.

A numerical method can be applied to find the sets of three variables. Fig. 9 shows a sample of these variables for a multiMW wind turbine at 8 different rotor speeds. The line with dots represents the steady state operating conditions for producing the rated power, i.e. $\left(v_{o}, \Omega_{r o}, \beta_{o}\right)$, at the rated rotor speed. The 5 lines above this line show the steady state operating conditions for 5 rotor speeds below the rated value, while the two lines below the line with dots are for 2 rotor speeds above the rated value. The relationship between the set of variables, $\left(v_{o}, \Omega_{r o}, \beta_{o}\right)$, shown in Fig. 9 can be expressed as:

$$
\beta_{o}=f\left(v_{o}, \Omega_{r o}\right)
$$

Using the above relationship, the amount of pitch, $\delta \beta_{F F}$, which is needed to maintain the rated power for a change of wind speed, $\delta v$ and the rotor speed, $\delta \Omega_{r}$ from the steady state, can be calculated as follows:

$$
\begin{aligned}
& \beta_{0}+\delta \beta_{F F} \simeq f\left(v_{0}, \Omega_{r_{0}}\right)+\left(\frac{\partial f}{\partial v}\right)_{o} \delta v+\left(\frac{\partial f}{\partial \Omega_{r}}\right)_{o} \delta \Omega_{r} \\
& \Rightarrow \delta \beta_{F F}=\left(\frac{\partial f}{\partial v}\right)_{o} \delta v+\left(\frac{\partial f}{\partial \Omega_{r}}\right)_{o} \delta \Omega_{r}
\end{aligned}
$$

The $\delta \beta_{F F}$ in the above equation is the feedforward amount in Fig. 8, which is the feedforward command to the pitch actuator.

The two feedforward gains in Eq. (13), i.e. $(\partial f / \partial v)_{0}$ and $\left(\partial f / \partial \Omega_{r}\right)_{o}$ are the same gains as $(\partial \beta / \partial v)_{o}$ and $\left(\partial \beta / \partial \Omega_{r}\right)_{o}$ from Fig. 8, respectively. The gains for each operating condition can be calculated numerically using the relationship in Fig. 9.

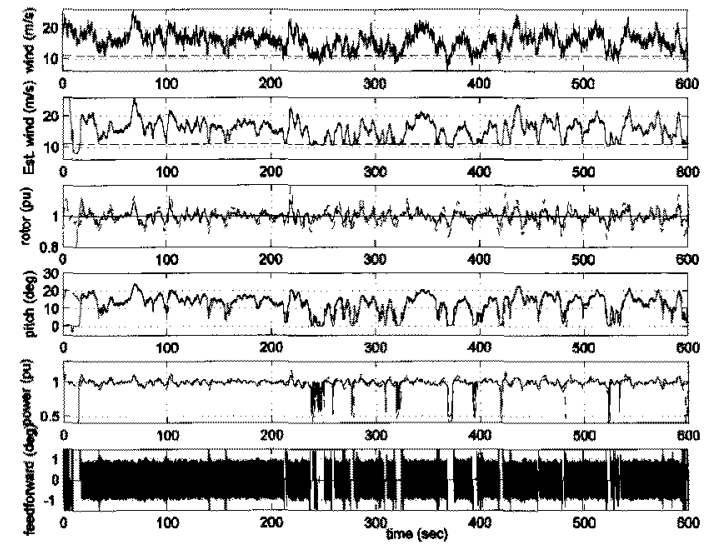

Fig. 10. Time domain responses of applying the feedforward control for the mean wind speed of $16 \mathrm{~m} / \mathrm{s}$ and $18 \%$ turbulence intensity.

\section{VERIFICATION THROUGH THE NUMERICAL SIMULATION}

The performance of the feedforward pitch control was estimated by numerical simulations. Fig. 10 shows the simulation results. The same PI pitch controller and torque schedule as those used for the simulation in Fig. 7 were applied. The dashed plots of Fig. 10 represent the responses of the wind turbine when the feedforward control is off (i.e. the responses shown as dashed lines are the same as those of Fig. 7), while the solid ones are the responses when it is on. The estimation of the aerodynamic torque was based on the Kalman filter of Eq. (9). In this calculation, $1 \%$ of the rated rpm and $8 \%$ of the rated torque are assumed as the measurement and the process noise, respectively. The large excursions in the responses during the initial stage of the simulation are due to the mismatched initial conditions of the estimator. The first window of Fig. 10 shows the wind used in this simulation, which is the same as that from Fig.7, i.e. a mean wind speed of $16 \mathrm{~m} / \mathrm{s}$ and $18 \%$ TI. The second window is the estimated wind speed, which is reconstructed using the 3-diemsioanl table from Fig. 8. Note that there is a slight time delay between the real and estimated wind and that the high frequency components in the turbulent wind are filtered out. The third window shows the rotor speed in units of PU. Compared to the response when the feedforward is off, which is represented by the dashed line, the fluctuation of the rotor speed over the rated value is decreased significantly. The fourth and the fifth windows are the plots of the pitch angle and the output power. The final window shows the amount of the feedforward control demand for the pitch actuation system.

It is a little difficult to discern the advantage of the feedforward control in these responses because the two responses are overlapped. In order to look more closely at the differences between these responses, the responses of Fig 10 from 130 seconds to 230 are re-plotted in Fig. 11. The efficacy of applying the feedforward control is clearly visible in this figure. Note again a sudden increase in the wind speed at around 215 seconds. In the case of not using the feedforward control, there is a large excursion of the rotor speed and the power. However, the time-ahead feedforward pitch control action minimizes the rotor speed fluctuations over the rated rpm. This is manifest in the pitch response at around 215 


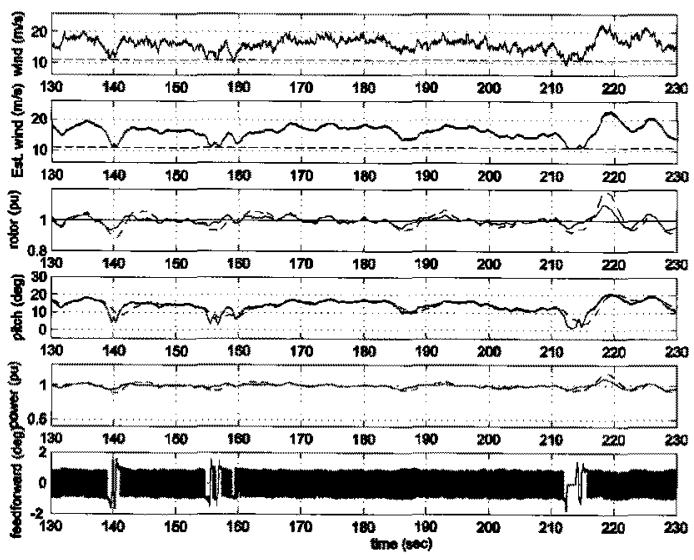

Fig. 11. Re-plot of Fig. 10 for the time period from 130 to 230 seconds.

TABLE II

Performance Data Comparing FeEdforward ON/OFF CONTRol

\begin{tabular}{|c|c|c|c|}
\hline \multicolumn{2}{|c|}{ Performance data } & Feedforward on & Feedforward off \\
\hline \multirow{2}{*}{$\begin{array}{c}\text { Rotor } \\
\text { (pu) }\end{array}$} & Mean & 1.0015 & $1.00 \overline{33}$ \\
\cline { 2 - 4 } & Std. deviation & 0.0299 & 0.0518 \\
\hline $\begin{array}{c}\text { Power } \\
\text { (pu) }\end{array}$ & Mean & 0.9962 & 0.9979 \\
\cline { 2 - 4 } & Std. deviation & 0.0298 & 0.0515 \\
\hline
\end{tabular}

seconds. Note that the pitch response for the feedforwardon (the solid line) is always leading the response for the feedforward-off (the dashed line). Similar feedforward actions can be seen in the responses of Fig. 11 at around 140 and 156 seconds. The performance enhancement from applying the feedforward pitch control is summarized in Table II. The standard deviations of the rotor speed or the power when the feedforward control is on, are approximately $50 \%$ lower than when it is off.

Compared to the pitch response when not applying the feedforward control, the high frequency components in the feedforwad pitch response increase noticeably. This is due to the high frequency which is generated in calculating $\delta v$ based on the differences in samples, i.e. $\delta v=v\left(t_{k}\right)-v\left(t_{k-1}\right)$ for a sampling rate of $100 \mathrm{~Hz}$. A low pass filter can be used to attenuate these high frequency components in the pitch demand signal. Even though simulation results are not included in this paper, somewhat similar results are obtained for the case of applying a low pass filter to the feedforward pitch demand.

\section{Conclusions}

A feedforward pitch control to enhance the performance characteristics of a multi-MW wind turbine is investigated. It turns out that the standard deviations of the rotor speed and the electric power over the rated value decrease approximately $50 \%$ from those obtained when not applying the feedforward control. However, this conclusion is based on a drive train model which is suitable only for a performance analysis of the wind turbine. The benefits of the feedforward control are further investigated from the point of view of performance and mechanical loads by using a more sophisticate aero-elastic simulation tool. Another point is the increase in the duty cycles of the pitch actuators for compensating wind speed variations, which should be scrutinized more in an aero-elastic simulation.

\section{ACKNOWLEDGMENT}

This work was supported by a grant from the Human Resources and New \& Renewable Energy division of the Korea Institute of Energy Technology Evaluation and Planning (KETEP) funded by the Ministry of Knowledge Economy, Republic of Korea (Grant No. 2008NBLHME080000 and 20093021020030).

\section{REFERENCES}

[1] T, Petru and T, Thiringer, "Modeling of wind turbines for power system studies," IEEE Trans, on Power Syst., Vol. 17, No. 4, pp. 1132-1139, Nov. 2002.

[2] F. D. Bianchi, H. D. Battisa, and R. J. Mantz, Wind turbine control systems, principles, modeling and gain scheduling design, Springer, 2007.

[3] J. Manwell, J. McGowan, and A. Rogers, Wind energy explained, Theory, design and application, A John Wiley and Sons, 2009.

[4] E. van der Hooft and T. van Engelen, Feedforward control of estimated wind speed, ECN-C-03-137, 2003.

[5] S. Muller, M. Deicke, and R. de Donker, "Doubly fed induction generator systems for wind turbines," IEEE Industry Applications Magazine, Vol. 8, No. 3, pp. 26-33, May/Jun. 2002.

[6] M. Chinchilla, S. Arnaltes, and J. C. Burgos, "Control of permanentmagnet generators applied to variable-speed wind energy systems connected to grid," IEEE Trans. on Energy Conversion, Vol. 21, No. 1, pp. 130-135, Mar. 2006.

[7] H.-G. Park, D.-C. Lee, and H.-G. Kim, "Cost-effective converters for micro wind turbine system using PMSG,"Joumal of Power Electronics, Vol. 8, No. 2, pp.156-162, Apr. 2008

[8] H.-S. Ko, G.-G. Yoon, and W.-P. Hong, "Active Use of DFIG-Based Variable-Speed Wind-Turbine for Voltage Control in Power System Operation," Journal of Electrical Engineering \& Technology, Vol 3, No 2, pp.254-262, Jun. 2008.

[9] E. van der Hooft, P. Schaak, and T. van Engelen, Wind turbine control algorithm, ECN-C-03-11, 2003.

[10] W. Leithead and B. Connor, "Control of variable speed wind turbines: dynamic models," International Journal of Control, Vol. 73, No. 13, pp. 1173-1188, Sep. 2000.

[11] S. Dominguez and W. Leithead, "Size related performance limitations of wind turbine control performance," in Proc. ICC, Glasgow, FB4-181, Sep. 2006.

[12] A. Gelb, Applied optimal estimation, The MIT Press, 1974.

[13] K. Ostergaard, P. Brath, and J. Stoustrup, "Estimation of effective wind speed," Journal of Physics: Conference series, Vol. 75, pp. 1-9, 2007.

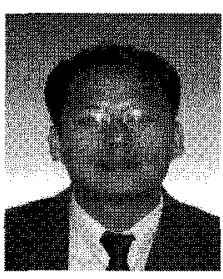

Yoonsu Nam received his B.S. in Nuclear Engineering and his M.S. in Mechanical Engineering from Seoul National University, Republic of Korea, in 1981 and 1983, respectively. He received his Ph.D. in Mechanical Engineering from the Georgia Institute of Technology, Atlanta, in 1991, where he worked on the tip position control of a flexible manipulator using a proof mass. From 1992 to 1996 , he was with the Flight Dynamics and Control Laboratory of the Agency for Defense Development, Korea. He is currently a Professor in the Mechanical and Mechatronics Engineering Department, Kangwon National University, Republic of Korea. His main interest is the design of wind turbine control systems.

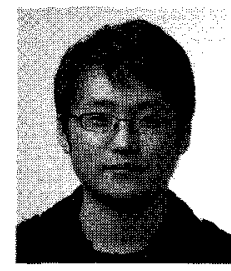

Jeonggi Kim received his B.S. and M.S. in Mechanical and Mechatronics Engineering from Kangwon National University, Chuncheon, Republic of Korea, in 2009 and 2011, respectively. He is currently working for the Control \& Monitoring Laboratory at Kangwon National University. His research interests are in the design of wind turbine control systems, load calculation and the evaluation of wind turbine systems. 


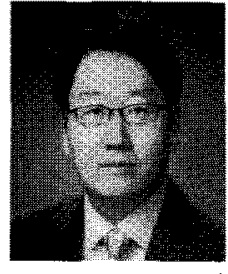

Insu Paek received his B.S. in Mechatronics Engineering from Kangwon National University, Chuncheon, Republic of Korea, in 1997, and his M.S. in Mechanical Engineering from the University of Texas at Austin, USA, in 2000. He received his Ph.D. in Mechanical Engineering from Purdue University, Indiana, USA, in 2005. He worked as a Postdoctoral Researcher at Purdue University and at Mcgill University, Quebec, Canada, in 2006 and 2007. He is currently an Assistant Professor in the Department of Mechanical and Mechatronics Engineering, Kangwon National University, Republic of Korea. His research interests include thermoacoustic cooling, solar powered absorption cooling and wind power generation.

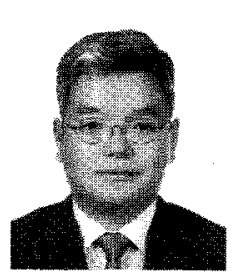

Young-Hwan Moon was born in Incheon, Republic of Korea, in 1956. He received his B.S. and M.S. from Seoul National University, Republic of Korea in 1979 and 1981 respectively, and his Ph.D. from the University of Texas at Arlington in 1990, all in Electrical Engineering. Since 1981, he has been with the Korea Electrotechnology Research Institute (KERI), where he is currently the Director of the Smart Grid Research Center. He current research interests include power system modeling from tests, power system stabilizers, and wide area measurement and control systems. He is a member of IEEE and KIEE.

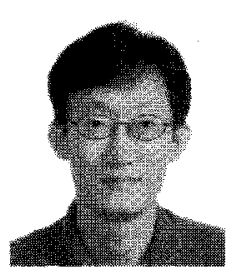

Seog-Joo Kim received his B.S. and M.S. in Electrical Engineering, and his Ph.D. in Electrical and Electronic Engineering from Yonsei University, Republic of Korea, in 1984, 1986, and 2007, respectively. Since 1986, he has been working for the Korea Electrotechnology Research Institute (KERI), where he is currently a Principal Researcher in the Smart Grid Research Center. His research interests include digital controller impleand control mentation, robust control, and power system protection

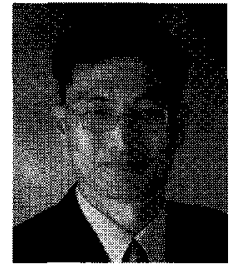

and IEEE
Dong-Joon Kim was born in the Republic of Korea, on January 7, 1971. He received his B.S., M.S., and Ph.D. in Electrical Engineering from Chonnam National University, Republic of Korea, in 1992, 1994, and 2004, respectively. He has been with KERI since 1994 and is now a Senior Research Engineer. His interests include the analysis of voltage stability, dynamic modeling of power plants, control of HVDC modeling, and the application of digital PSS. He is a Member of KIEE 